\title{
A PRODUÇÃO CIENTÍFICA SOBRE $O$ JIU-JITTSU: ANÁLISE DOS ARTIGOS, TESES E DISSERTAÇÕES PUBLICADOS ENTRE 1996 E 2016
}

\author{
SCIENTIFIC PRODUCTION ON JIU-JITTSU: ANALYSIS OF ARTICLES, THESES \\ AND DISSERTATIONS PUBLISHED BETWEEN 1996 AND 2016
}

\author{
LA PRODUCCIÓN CIENTIIFICA SOBRE EL JIU-JITTSU: ANÁLISIS DE LOS \\ ARTÍCULOS, TESIS Y DISERTACIONES PUBLICADOS ENTRE 1996 Y 2016
}

\author{
Lucas Vicentini ${ }^{\star}$ Renato Francisco Rodrigues Marques*
}

\section{Palavras chave:}

Jiu-jítsu.

Produção científica.

Bibliometria.

Pesquisa.
Resumo: 0 objetivo deste trabalho foi analisar e descrever a produção científica relacionada ao jiu-jítsu, de modo a identificar tendências, lacunas e possibilidades de desenvolvimento de pesquisa. Através de busca nas bases de dados digitais Web of Science, SciELO, Scopus, PubMed, Bireme, LILACS e Redalyc, além do banco de teses e dissertações da Capes, e análise temática de 105 artigos, duas teses e 23 dissertações, tem-se como principais resultados: a) desigualdade numérica entre diferentes abordagens e subáreas de pesquisa, bem como disciplinas de estudo; b) característica heterogênea dos participantes destes estudos, sendo a maioria homens adultos não competidores; c) destaque do Brasil na produção científica sobre o jiu-jítsu; d) crescimento quantitativo e qualitativo de pesquisas; e) processo de internacionalização de pesquisas. Conclui-se que, assim como no âmbito esportivo mais amplo, a pesquisa sobre jiu-jítsu se encontra em franca expansão, principalmente no Brasil, com lacunas que representam potenciais áreas de interesse para investigações futuras.

\section{Keywords:}

Jiu-Jitsu.

Academic

production.

Bibliometrics.

Research.

\begin{abstract}
The aim of this article was to analyse and describe the scientific production related to jiu-jitsu, in order to identify trends, gaps and research possibilities. Searches on the digital data basis Web of Science, SciELO, Scopus, PubMed, Bireme, LILACS and Redalyc, besides Capes's digital thesis library, and thematic analysis on 105 articles, 2 theses and 23 dissertations, yielded the following main results: a) numerical inequality between different research approaches and subareas, as well as study subjects; $b$ ) the profiles of participants in those studies are highly diverse, and most are male non-competitors; c) Brazil stands out in academic production on jiu-jitsu; d) increasing quantitative and qualitative research; e) process of internationalization of research. The study found that, just as in the sports field as a whole, research on jiu-jitsu is in full expansion, mainly in Brazil, with gaps that are potential areas of interest for future investigation.
\end{abstract}

Palabras clave: Jiu-jitsu. Producción científica. Bibliometría. Investigación.
Resumen: El objetivo de este trabajo fue analizar y describir la producción científica relacionada con el jiu-jitsu, para identificar tendencias, lagunas y posibilidades de desarrollo de investigación. A través de la búsqueda en las bases de datos digitales Web of Science, SciELO, Scopus, PubMed, Bireme, LILACS y Redalyc, además del banco de tesis y disertaciones de Capes, y del análisis temático de 105 artículos, dos tesis y 23 disertaciones, se tienen como principales resultados: a) desigualdad numérica entre diferentes aproximaciones y subáreas de investigación, así como disciplinas de estudio; b) característica heterogénea de los participantes en estos estudios, donde la mayoría es de hombres adultos no competidores; c) destaque de Brasil en la producción científica sobre el jiu-jitsu; d) crecimiento cuantitativo y cualitativo de investigaciones; e) proceso de internacionalización de investigaciones. Se concluye que, así como en el ámbito deportivo más amplio, la investigación sobre jiu-jitsu se encuentra en expansión, principalmente en Brasil, con lagunas que representan potenciales áreas de interés para investigaciones futuras.
* Universidade de São Paulo. São Paulo, SP, Brasil.

E-mail: lucas.vicentini@usp.br: renatomarques@usp.br

Recebido em: 07/06/2018 Aprovado em: 29/10/2018

DOI: https://doi.org/10.22456/1982-8918.83697 (c) (1) () Licence 


\section{INTRODUÇÃO}

Durante a segunda metade do século XX, e principalmente nas últimas décadas, algumas artes marciais orientais experimentaram processos de esportivização e deram origem a modalidades esportivas de combate. Uma delas é o jiu-jítsu brasileiro, herdeiro do antigo ju-jítsu de origem japonesa, que teve o Brasil como cenário de transformação e consolidação (GREEN; SVINTH, 2010).

A relevância da vertente brasileira do jiu-jítsu pode ser atestada por ser praticada inclusive no Japão, o que estabelece os termos jiu-jítsu, jiu-jítsu brasileiro e jiu-jítsu Gracie como sinônimos (PAIVA, 2009), sendo retratado no Brasil e neste trabalho unicamente como jiu-jítsu.

Através principalmente da grande exposição midiática proporcionada pelo espetáculo do Mixed Martial Arts (MMA), o jiu-jítsu alcançou projeção internacional (GREEN; SVINTH, 2010), com suas competições reunindo atletas de diferentes partes do mundo (INTERNATIONAL BRAZILIAN JIU-JITSU FEDERATION, 2018), tendo os brasileiros um grande destaque. Neste contexto, o ambiente de alto rendimento do jiu-jítsu vive um processo lento e gradativo de estabelecimento de profissionalização (VICENTINI; MARQUES, 2018). O jiu-jítsu se encontra em franco crescimento, tanto em visibilidade, quanto no número de praticantes, demonstrando relevância no mercado esportivo (PAIVA, 2009) e despertando interesse do campo acadêmico (ANDREATO et al., 2016; DIAZ-LARA et al., 2015; KREISWIRTH; MYER; RAUH, 2014; LISE; SANTOS; CAPRARO, 2014).

No Brasil, as lutas, artes marciais e modalidades esportivas de combate (L/AM/MEC), incluindo o jiu-jítsu, contam com um campo de estudos ainda jovem, que já tem contribuído com pesquisas promissoras (BRAUER et al., 2014; CORREIA; FRANCHINI, 2009; FRANCHINI; DEL VECCHIO, 2011). Portanto, a pergunta central deste trabalho foi: como se caracteriza a produção científica relacionada ao jiu-jítsu?

O objetivo geral foi caracterizar e descrever a produção científica relacionada ao jiu-jítsu, de modo a identificar tendências, lacunas e possibilidades de desenvolvimento de pesquisa. Como objetivos específicos têm-se:

a) analisar artigos indexados em importantes bases de dados digitais;

b) analisar dissertações e teses produzidas no Brasil;

c) identificar as abordagens de pesquisa, subáreas e disciplinas de estudo;

d) caracterizar os participantes dos estudos;

e) mapear a distribuição geográfica de pesquisadores;

f) caracterizar os periódicos onde foram veiculadas as publicações;

g) identificar os idiomas de divulgação acadêmica;

h) identificar a distribuição cronológica das pesquisas.

\section{MÉTODOS}

Com o crescimento global da produção e divulgação de material científico, é crescente também a necessidade de mapear as características de diferentes campos do conhecimento. Sob essa justificativa, se fundamenta a bibliometria, que, segundo Araújo (2006), visa 
quantificar, classificar e mapear a produção e disseminação de conhecimento científico sobre determinado assunto. Esta análise, portanto, promove reflexões pertinentes aos interessados pelo jiu-jítsu, através da identificação de objetos de estudos frequentes, tendências, lacunas ou até mesmo contradições na literatura.

A Análise Temática (BRAUN; CLARKE, 2006) foi utilizada para qualificar esses trabalhos sobre diferentes temas, sendo estes posteriormente quantificados, com a intenção de identificar tendências e lacunas sobre estudos relacionados ao jiu-jítsu.

\subsection{Coleta de dados}

A primeira etapa desta investigação foi a consulta às bases de dados digitais indexadoras de importantes periódicos da área de Educação Física e Esporte: Web of Science, SciELO, Scopus, PubMed, Bireme, LILACS e Redalyc. Para o acesso a teses e dissertações produzidas no Brasil, foi consultado o Banco de Teses do Portal da Coordenação de Aperfeiçoamento de Pessoal de Nível Superior (Capes). A análise dos estudos se deu a partir do ano de 1996, quando a Capes começou a disponibilizar o catálogo de dissertações e teses, até dezembro de 2016. Para tal, foram utilizados os termos de busca: "jiu-jítsu", "jiu jítsu" ou "jiujítsu".

\subsection{Análise dos dados}

O método Análise Temática foi utilizado para identificar, analisar e relatar padrões (temas) dentro de um corpo de dados, de acordo com o interesse particular delineado pelos objetivos do estudo (BRAUN; CLARKE, 2006). Tais dados foram oriundos de análise sobre os títulos, resumos, palavras-chave e informações bibliográficas dos trabalhos selecionados.

Por vezes, a informação contida nos resumos (principal conjunto de dados analisados) foi limitada, pois sua escrita é regida por normas heterogêneas que podem desconsiderar a complexidade dos trabalhos representados (FERREIRA, 2002). A exemplo de Abreu, Fernandes e Martins (2009), foi realizada a leitura do texto completo, ou em partes, quando 0 resumo ofereceu dados insuficientes para a investigação proposta.

A Figura 1 mostra as etapas da Análise Temática aplicadas neste estudo.

Figura 1 - Etapas da Análise Temática.

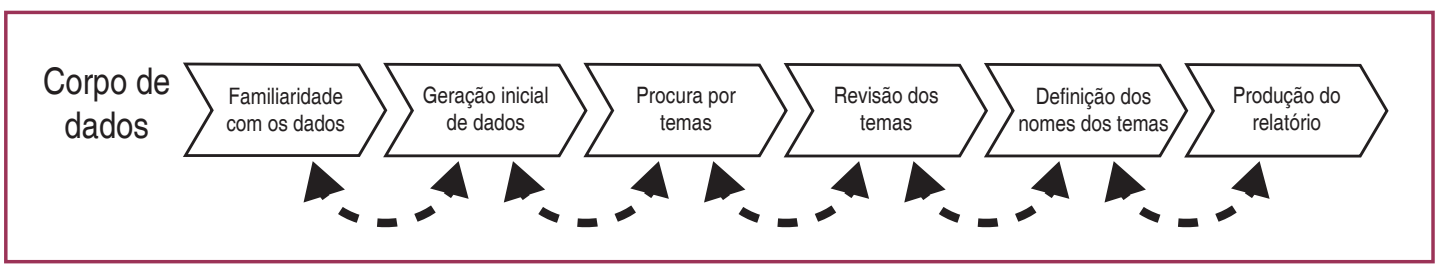

Fonte: Adaptado de Braun e Clarke (2006).

As setas duplas na Figura 1 significam que entre as etapas da Análise Temática não existe uma ordem inflexível, mas, sim, uma construção por parte do pesquisador. O método permite que o pesquisador volte e avance em suas etapas enquanto trabalha seus dados, com base no seu objetivo e referencial teórico. 
Na etapa de "familiarização com os dados", foi realizada a leitura prévia do título, resumo e palavras-chave de todas as publicações catalogadas, tendo as informações bibliográficas relacionadas. Esta primeira leitura permitiu uma visão geral do corpo de dados, a partir da qual foram geradas as primeiras impressões importantes para as fases posteriores. Nesta etapa, foram identificados os trabalhos que, apesar de trazer em seu título, resumo ou palavras chave os termos de busca utilizados, não tinham o jiu-jítsu como objeto de pesquisa.

A etapa de "geração inicial de dados" se deu quando todo o corpo de dados resultante da primeira triagem foi disposto no instrumento de análise. O material foi relido cuidadosamente, sendo extraídos dados relevantes à temática expressa nos objetivos desta pesquisa. Nesta fase, foram gerados os códigos iniciais para classificar os dados extraídos, sendo, ao término deste processo, agrupados em conjunto dentro de cada código, sem perder a identificação com 0 documento de origem.

$\mathrm{Na}$ etapa subsequente, denominada "procura por temas", os códigos propostos e todos os dados que os abrangem foram organizados em temas potenciais de acordo com os objetivos desta pesquisa. Os temas foram revisados, visando ao seu aperfeiçoamento na etapa de "revisão dos temas". Temas sustentados por dados insuficientes ou incoerentes foram mesclados, redefinidos ou excluídos.

No final desse processo, se fez necessária a "definição dos nomes dos temas", refinando as especificidades de cada um.

Por fim, na "produção do relatório", os temas foram dispostos, buscando dar fluidez à discussão proposta neste trabalho. Nessa etapa, a quantificação dos temas se deu como um complemento para oferecer informações pertinentes à discussão.

\section{RESULTADOS}

As pesquisas nas bases de dados resultaram em 80 (Scopus), 73 (Bireme), 62 (Web of Science), 35 (PubMed), 31 (LILACS), 17 (SciELO) e 11 (Redalyc) artigos. Trabalhos duplicados foram excluídos, totalizando um corpo de dados com 122 artigos.

A pesquisa no banco de teses e dissertações do portal Capes resultou em três teses de doutorado e 33 dissertações de mestrado, das quais foram excluídas da análise dez dissertações que não tiveram os arquivos disponibilizados na íntegra para download, totalizando um corpo de dados com três teses e 23 dissertações.

Através da etapa de familiarização dos dados, foi possível identificar uma tese e 17 artigos que, apesar de citarem um dos termos pesquisados em seu título, resumo ou palavraschave, não se referiam ao jiu-jítsu, a exemplo de trabalhos voltados ao MMA, que citam o jiujítsu em trechos introdutórios de seus resumos (BLEDSOE et al., 2006). Esses trabalhos foram retirados, resultando em duas teses, 23 dissertações e 105 artigos conforme esquematizado na Figura 2.

Estes trabalhos restantes foram analisados através das etapas seguintes da Análise Temática, com base em categorias de análise, de acordo com os objetivos deste trabalho: Abordagem de pesquisa; Subárea de pesquisa e disciplina de estudo; Caracterização dos participantes dos estudos; Análise geográfica; Análise por periódicos; Idioma das publicações; Análise cronológica da pesquisa. 
Figura 2 - Representação esquemática da busca por artigos, teses e dissertações, e estruturação do corpo de dados.

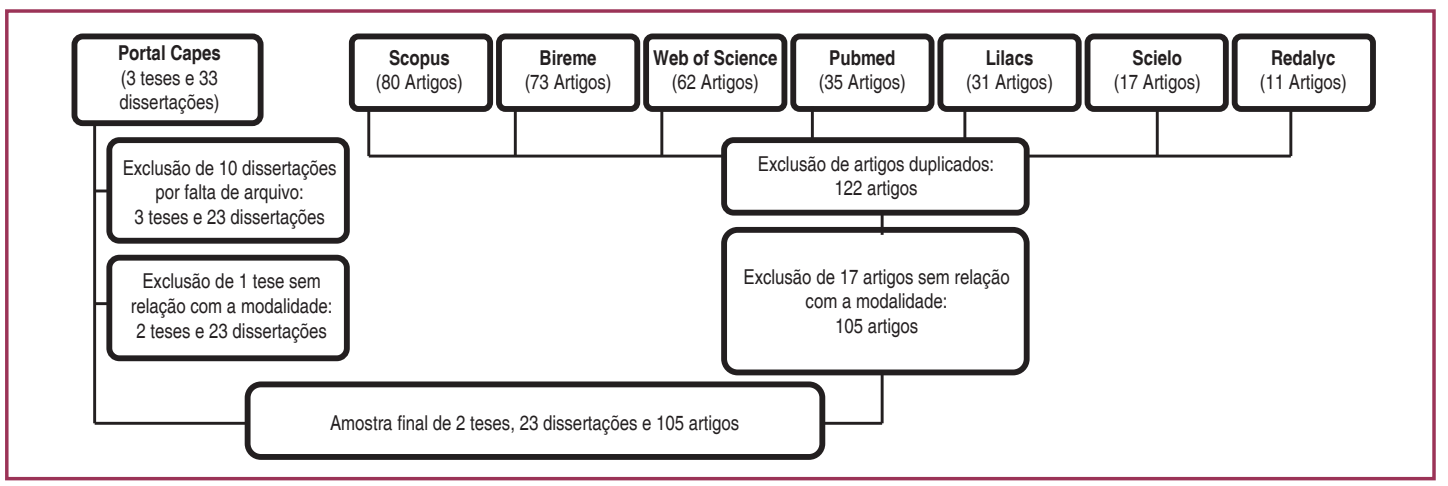

Fonte: os autores

\subsection{Abordagem de pesquisa}

Compreender a distribuição dos trabalhos de acordo com as abordagens de pesquisa se faz pertinente para conhecer o cenário da pesquisa sobre determinado objeto (AQUINO et al. 2017). Os diferentes problemas estudados na atividade física e esporte implicam diferentes abordagens de pesquisa (THOMAS; NELSON; SILVERMAN, 2007). Dentre as classificações existentes, optou-se por distinguir as pesquisas de ordem quantitativa e qualitativa. Tais abordagens podem se complementar (MINAYO; SANCHES, 1993). Neste estudo, pesquisas que abordam as duas formas foram classificadas como quantitativo-qualitativas.

Através da Análise Temática, identificou-se que 76,2\% dos trabalhos do corpo de dados tratam de pesquisas quantitativas, $16,2 \%$ qualitativas e os outros $7,7 \%$ quantitativoqualitativas. A Tabela 1 apresenta a análise das abordagens de pesquisa expostas nos artigos, teses e dissertações analisadas.

Tabela 1-Abordagens de pesquisa com o jiu-jítsu.

\begin{tabular}{lccc}
\hline Abordagem de pesquisa & Artigos & Teses e dissertações & Corpo de dados (total) \\
\hline Quantitativa & $83(79 \%)$ & $16(64 \%)$ & $99(76,2 \%)$ \\
Qualitativa & $13(12,4 \%)$ & $8(32 \%)$ & $21(16,2 \%)$ \\
Quantitativo-qualitativa & $9(8,6 \%)$ & $1(4 \%)$ & $10(7,7 \%)$ \\
\hline Total & $105(100 \%)$ & $25(100 \%)$ & $130(100 \%)$ \\
\hline
\end{tabular}

Fonte: os autores.

\subsection{Subáreas de pesquisa e disciplinas de estudo}

Foi possível classificar os trabalhos de acordo com a subárea de pesquisa adotada e a disciplina de estudo relacionada ao jiu-jítsu.

A presente análise dividiu o corpo de dados em duas subáreas, "biodinâmica" e "sociocultural e pedagógica", representando respectivamente $80 \%$ e $20 \%$ do corpo de trabalhos analisados. A subárea biodinâmica é formada por linhas de pesquisa básica ou aplicada, orientadas pelas ciências naturais (MANOEL; CARVALHO, 2011), enquanto a subárea sociocultural e pedagógica se relaciona à pesquisa que desenvolve temáticas em interface com as ciências humanas e sociais (CARNEIRO et al., 2016). 
As disciplinas de estudo foram subdivisões observadas dentro de cada subárea de pesquisa. A classificação por disciplina permite observar a conformação do cenário da pesquisa acadêmica sobre o jiu-jítsu com maiores detalhes. Os estudos relacionados à biodinâmica foram classificados em "Fisiologia do exercício" (29,2\%); "Traumatologia" (16,2\%); "Biomecânica" (12,3\%); "Nutrição esportiva" (12,3\%); "Avaliação da Aptidão Física" (9,2\%) e "Esporte adaptado/fisiologia" (1\%). Já a subárea Sociocultural e pedagógica foi composta pelas disciplinas "História do esporte" (6,2\%); "Sociologia do esporte" (6,2\%); "Psicologia do esporte" $(5,4 \%)$ e "Pedagogia do esporte" (2,3\%).

As tabelas 2 e 3 trazem respectivamente a análise completa em relação à subárea de pesquisa e às disciplinas de estudo dos artigos e das teses e dissertações envolvendo o jiujítsu.

Tabela 2 - Subáreas de pesquisa do jiu-jítsu.

\begin{tabular}{lccc}
\hline \multicolumn{1}{c}{ Subárea de pesquisa } & Artigos & Teses e dissertações & Corpo de dados (total) \\
\hline Biodinâmica (SB) & $87(82,9 \%)$ & $17(68 \%)$ & $104(80 \%)$ \\
Sociocultural e pedagógica (SSP) & $18(17,1 \%)$ & $8(32 \%)$ & $26(20 \%)$ \\
\hline Total & $105(100 \%)$ & $25(100 \%)$ & $130(100 \%)$ \\
\hline
\end{tabular}

Fonte: os autores.

Tabela 3 - Disciplinas de estudo do jiu-jítsu.

\begin{tabular}{|c|c|c|c|c|}
\hline & Disciplinas de estudo & Artigos & Teses e dissertações & Corpo de dados (total) \\
\hline \multirow{6}{*}{$\stackrel{\mathscr{M}}{\mathscr{N}}$} & Fisiologia do exercício* & $31(29,5 \%)$ & $7(28 \%)$ & $38(29,2 \%)$ \\
\hline & Traumatologia & $18(17,1 \%)$ & $3(12 \%)$ & $21(16,2 \%)$ \\
\hline & Biomecânica & $13(12,4 \%)$ & $3(12 \%)$ & $16(12,3 \%)$ \\
\hline & Nutrição esportiva & $14(13,3 \%)$ & $2(8 \%)$ & $16(12,3 \%)$ \\
\hline & Avaliação da aptidão física & $10(9,5 \%)$ & $2(8 \%)$ & $12(9,2 \%)$ \\
\hline & Esporte adaptado/fisiologia** & $1(1 \%)$ & $0(0 \%)$ & $1(1 \%)$ \\
\hline \multirow{5}{*}{$\begin{array}{l}\text { 怘 } \\
\text { क }\end{array}$} & História do esporte & $7(6,7 \%)$ & $1(4 \%)$ & $8(6,2 \%)$ \\
\hline & Sociologia do esporte & $3(2,9 \%)$ & $5(20 \%)$ & $8(6,2 \%)$ \\
\hline & Psicologia do esporte & $7(6,7 \%)$ & $0(0 \%)$ & $7(5,4 \%)$ \\
\hline & Pedagogia do esporte & $1(1 \%)$ & $2(8 \%)$ & $3(2,3 \%)$ \\
\hline & Total & $105(100 \%)$ & $25(100 \%)$ & $30(100 \%)$ \\
\hline \multicolumn{5}{|c|}{$\begin{array}{l}\text { Fonte: os autores. } \\
\text { * Quatro trabalhos, sendo três artigos e uma dissertação relacionados à "fisiologia do exercício" contribuíram também com análises técnico- } \\
\text { táticas em situação de combate. } \\
\text { * } O \text { único trabalho que investigou o jiu-jítsu no contexto do esporte adaptado foi desenvolvido por Matos-Souza et al. (2016), com o objetivo } \\
\text { de avaliar o impacto da prática do esporte adaptado (jiu-jítsu, rúgbi, basquetebol e tênis) no quadro de aterosclerose em sujeitos com lesão na } \\
\text { medula espinhal. Dada à abrangência da área de investigação dos esportes adaptados, é importante sinalizar que o referido trabalho se situa } \\
\text { na subárea biodinâmica, tendo a fisiologia como disciplina de estudo. }\end{array}$} \\
\hline
\end{tabular}

\subsection{Caracterização dos participantes dos estudos}

Dentre os 130 trabalhos, foi identificada a participação de sujeitos envolvidos de alguma forma com o jiu-jítsu em 109. Os 21 trabalhos restantes consistiam em revisões de 
literatura, estudos baseados em documentos históricos e ensaios relacionados ao jiu-jítsu que não contavam com participantes. Deste grupo de 109, o jiu-jítsu dividiu o foco do estudo com uma a nove outras modalidades esportivas em 30 trabalhos (27,5\%), sendo 22 artigos, sete dissertações e uma tese.

Os participantes foram classificados em oito subtemas: "Praticantes não competidores (sem distinção por faixa)" (40,3\%); "Competidores não elite" (32,1\%); "Praticantes avançados não competidores (faixas marrom e preta)" (13,8\%); "Competidores de elite (nível nacional e internacional)" (7,3\%); "Instrutores, professores e mestres" (2,8\%); "Idosos" (1,8\%), "Pessoas com deficiência" (0,9\%) e "Adolescentes" (0,9\%).

A Tabela 4 apresenta a caracterização dos participantes nos estudos analisados.

Tabela 4 - Caracterização dos participantes dos estudos por tipo de envolvimento.

\begin{tabular}{lccc}
\hline \multicolumn{1}{c}{ Participantes da pesquisa } & Artigos & Teses e dissertações & $\begin{array}{c}\text { Corpo de dados } \\
\text { (total) }\end{array}$ \\
\hline Praticantes não competidores & $33(37,1 \%)$ & $11(55 \%)$ & $44(40,3 \%)$ \\
Competidores não elite & $30(33,7 \%)$ & $5(25 \%)$ & $35(32,1 \%)$ \\
Praticantes avançados não competidores & $14(15,7 \%)$ & $1(5 \%)$ & $15(13,8 \%)$ \\
Competidores de elite & $7(7,9 \%)$ & $1(5 \%)$ & $8(7,3 \%)$ \\
Instrutores, professores e mestres & $1(1,1 \%)$ & $2(10 \%)$ & $3(2,8 \%)$ \\
Idosos & $2(2,2 \%)$ & $0(0 \%)$ & $2(1,8 \%)$ \\
Pessoas com deficiência & $1(1,1 \%)$ & $0(0 \%)$ & $1(0,9 \%)$ \\
Adolescentes & $1(1,1 \%)$ & $0(0 \%)$ & $1(0,9 \%)$ \\
\hline Total & $89(100 \%)$ & $20(100 \%)$ & $109(100 \%)$ \\
\hline
\end{tabular}

Fonte: os autores.

Uma segunda análise permitiu identificar o perfil de gênero dos participantes destes 109 trabalhos, sendo 80,7\% exclusivamente do gênero masculino, 0,9\% do gênero feminino, e 18,3\% envolvendo participação de ambos (Tabela 5).

Tabela 5 - Caracterização dos participantes dos estudos por gênero.

\begin{tabular}{lccc}
\hline \multicolumn{1}{c}{ Gênero dos participantes } & Artigos & Teses e dissertações & Corpo de dados (total) \\
\hline Masculino & $72(80,9 \%)$ & $16(80 \%)$ & $88(80,7 \%)$ \\
Feminino & $1(1,1 \%)$ & $0(0 \%)$ & $1(0,9 \%)$ \\
Ambos & $16(18 \%)$ & $4(20 \%)$ & $20(18,3 \%)$ \\
\hline Total & $89(100 \%)$ & $20(100 \%)$ & $109(100 \%)$ \\
\hline \multicolumn{5}{c}{ Fonte: os autores. }
\end{tabular}

\subsection{Análise geográfica}

A análise da localização das instituições envolvidas em estudos com o jiu-jítsu permitiu observar a formação de polos de pesquisa e buscar compreender o cenário global da pesquisa científica envolvendo esta modalidade esportiva. 
Os 105 artigos analisados foram produzidos por pesquisadores representando 94 instituições nas Américas, Europa, Ásia e Oceania. Destas, 59 (62,8\%) estão situadas no Brasil, 16 (17\%) nos Estados Unidos e outras 20 distribuídas em mais onze países, sendo Canadá, Chile, Colômbia, Estônia, Espanha, Holanda, Polônia, Portugal, Reino Unido, Irã e Austrália. As três instituições em destaque na produção científica envolvendo o jiu-jítsu no mundo são brasileiras. A Universidade de São Paulo, a Universidade Estadual de Maringá e a Universidade Estadual de Santa Catarina foram centros geradores de, respectivamente, 25, 12 e dez artigos.

Os resultados indicaram também que $76(72,4 \%)$ trabalhos tiveram como primeiro autor pesquisadores representando instituições brasileiras, dez (9,5\%) dos Estados Unidos e cinco $(4,8 \%)$ da Espanha. Outros 14 (13,3\%) trabalhos foram dirigidos por pesquisadores representando instituições da Austrália, Canadá, Chile, Colômbia, Estônia, França, Holanda, Irã, Romênia e Polônia.

As 25 teses e dissertações produzidas no Brasil são oriundas de 19 instituições diferentes, distribuídas principalmente nas regiões Sudeste e Sul. Foram produzidos 14 (56\%) trabalhos na Região Sudeste, oito (32\%) na Região Sul, dois (8\%) na Região Centro-Oeste e um (4\%) na Região Nordeste. A Universidade Tecnológica Federal do Paraná destacou-se com três dissertações envolvendo o jiu-jítsu.

\subsection{Análise por periódicos}

Os 105 artigos envolvendo o jiu-jítsu foram publicados em 64 periódicos de 14 países das Américas, Europa e Ásia. O Brasil lidera com 24 (37,5\%) periódicos que já veicularam artigos sobre o jiu-jítsu, seguido por Estados Unidos e Reino Unido com $12(18,8 \%)$ e nove $(14,1 \%)$, respectivamente.

Os periódicos foram classificados primeiramente deacordo com seusestratos Webqualis na área 21 da Capes (Educação Física, Fisioterapia, Fonoaudiologia e Terapia Ocupacional), referentes ao quadriênio 2013-2016 (COORDENAÇÃO DE APERFEIÇOAMENTO DE PESSOAL DE NÍVEL SUPERIOR, 2018). Dos 64, 14 não tinham classificação Webqualis disponível na área 21 no momento da coleta de dados. Uma possibilidade para este evento acontecer é por serem trabalhos disponibilizados em periódicos em que nenhum pesquisador brasileiro vinculado à pós-graduação na área 21 tenha publicado anteriormente, com base na avaliação realizada no quadriênio de 2013-2016: "...se nenhum docente ou discente de um programa de pós-graduação credenciado tiver publicado um artigo naquela revista, não há nenhum sentido em incluí-la na lista" (BARATA, 2016, p.18).

Dado o fato desta classificação sempre ser feita a posteriori pela Capes, por vezes alguns periódicos podem deixar de ser incluídos também por problemas no processamento dos dados, em que, apesar de haver também uma classificação manual realizada por comissões a partir dos mesmos critérios adotados, reconhece-se por fim, que tal processo é sujeito a falhas, em que determinados periódicos podem sofrer com atrasos em sua promoção (BARATA, 2016).

Com o objetivo de procurar evidenciar os critérios para avaliação qualitativa de todos os periódicos onde foram vinculadas as publicações a respeito do jiu-jítsu, optou-se 
por realizar o exercício de proceder com tal classificação, tendo como base os critérios de classificação de periódicos da área 21 referentes ao mesmo quadriênio (COORDENAÇÃO DE APERFEIÇOAMENTO DE PESSOAL DE NÍVEL SUPERIOR, 2016). Essa prática é comum por parte das comissões de avaliação da Capes quando algum periódico não consta na lista do Webqualis: "Mesmo que, no último ano do período de avaliação, alguns periódicos não estejam incluídos como resultado de falhas no processamento dos dados, as comissões procederão à sua classificação manual, utilizando exatamente os mesmos critérios" (BARATA, 2016, p.18).

Sendo assim, sem nenhuma pretensão de assumir o papel dessas comissões, mas apenas em um exercício metodológico de reflexão específico para este trabalho, sobre uma possível classificação dos periódicos em questão (considerando que o objetivo deste trabalho é uma análise que inclua, mas que também extrapole o grupo dos artigos publicados por autores brasileiros), o resultado obtido possibilita uma tentativa de aproximação a uma eventual classificação do periódico, justificando assim o possível estrato Qualis dos 14 periódicos a partir da classificação realizada pelos autores deste trabalho, com base nos mesmos critérios adotados pela área 21 da Capes.

Nesta classificação, foram identificados respectivamente um, dois, cinco, três, dois e um periódicos que estariam classificados nos estratos $A 1, A 2, B 2, B 3, B 4$ e B5, respectivamente. Uma eventual desconsideração de tal classificação, ou a alocação desses trabalhos em um grupo denominado "não classificado no estrato Qualis", poderia distorcer o cenário qualitativo dos periódicos em que acontecem as publicações de trabalhos relacionados ao jiu-jítsu.

Dos 105 artigos, $15(14,3 \%)$ foram publicados em periódicos de estrato A1, $31(29,5 \%)$ em estrato A2 e 21 (20\%), 18 (17,1\%), $13(12,4 \%)$, seis (5,7\%) e um (1\%) em periódicos classificados em estratos B1, B2, B3, B4 e B5, respectivamente. Na Tabela 6 é apresentada a distribuição por estrato Qualis dos 64 periódicos que veicularam artigos sobre jiu-jítsu, além da relação dos 105 artigos, considerando que 18 periódicos possuíam mais de um trabalho sobre a modalidade esportiva em análise.

Tabela 6 - Distribuição por estrato Qualis considerando periódicos e artigos separadamente.

\begin{tabular}{ccc}
\hline Estrato & Periódicos & Artigos \\
\hline A1 & $13(20,3 \%)$ & $15(14,3 \%)$ \\
A2 & $11(17,2 \%)$ & $31(29,5 \%)$ \\
B1 & $13(20,3 \%)$ & $21(20 \%)$ \\
B2 & $11(17,2 \%)$ & $18(17,1 \%)$ \\
B3 & $9(14,1 \%)$ & $13(12,4 \%)$ \\
B4 & $6(9,4 \%)$ & $6(5,7 \%)$ \\
B5 & $1(1,6 \%)$ & $1(1 \%)$ \\
\hline Total & $64(100 \%)$ & $105(100 \%)$ \\
\hline
\end{tabular}

Fonte: os autores.

Em 18 periódicos, o jiu-jítsu foi temática recorrente entre dois e oito artigos. O Quadro 1 relaciona os resultados referentes à subárea de pesquisa e à disciplina de estudo dos artigos e periódicos. 
Quadro 1- Subáreas de pesquisa e disciplinas de estudo dos artigos publicados em periódicos com duas ou mais publicações sobre o jiu-jítsu.

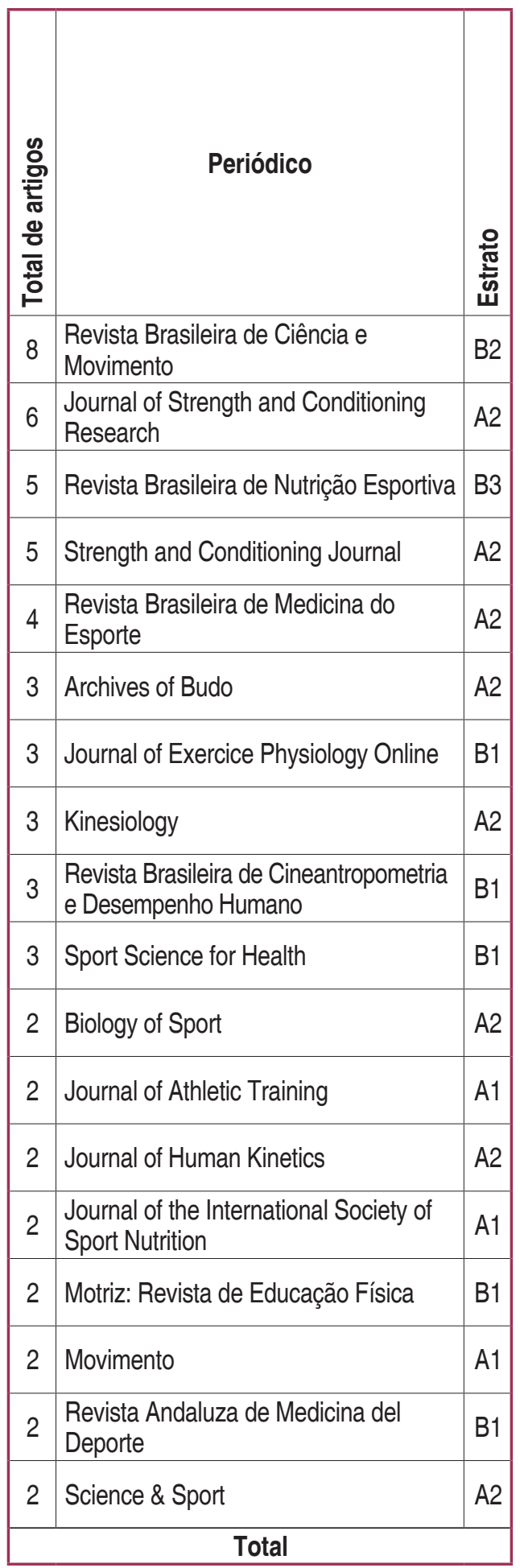

\begin{tabular}{|c|c|c|c|c|c|c|}
\hline 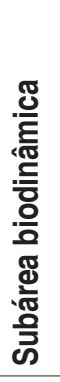 & 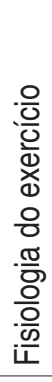 & 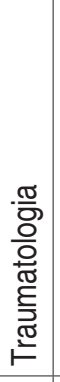 & 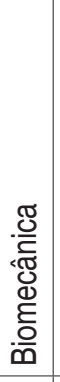 & 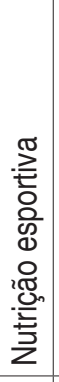 & 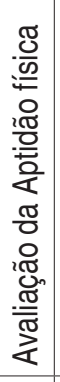 & 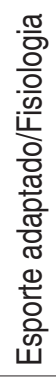 \\
\hline 6 & 1 & 0 & 1 & 2 & 2 & \\
\hline 6 & 4 & 4 & 1 & 0 & 0 & \\
\hline 5 & 0 & 0 & 0 & 5 & 0 & \\
\hline 5 & 5 & 0 & 0 & 0 & 0 & \\
\hline 4 & 0 & 2 & 2 & 0 & 0 & 0 \\
\hline 3 & 0 & 0 & 1 & 0 & 2 & 0 \\
\hline 3 & 2 & 0 & 0 & 0 & 1 & $v$ \\
\hline 3 & 3 & 0 & 0 & 0 & 0 & 0 \\
\hline 3 & 1 & 0 & 2 & 0 & 0 & 0 \\
\hline 3 & 1 & 0 & 0 & 0 & 2 & 0 \\
\hline 2 & 2 & 0 & 0 & 0 & 0 & 0 \\
\hline 2 & 1 & 1 & 0 & 0 & 0 & 0 \\
\hline 2 & 2 & 0 & 0 & 0 & 0 & 0 \\
\hline 2 & 0 & 0 & 0 & 2 & 0 & 0 \\
\hline 1 & 1 & 0 & 0 & 0 & 0 & 0 \\
\hline 0 & 0 & 0 & 0 & 0 & 0 & 0 \\
\hline 2 & 2 & 0 & 0 & 0 & 0 & 0 \\
\hline 2 & 1 & 0 & 0 & 0 & 1 & 0 \\
\hline 54 & 26 & 4 & 7 & 9 & 8 & 0 \\
\hline
\end{tabular}

\begin{tabular}{|c|c|c|c|c|}
\hline 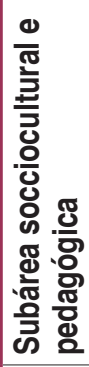 & 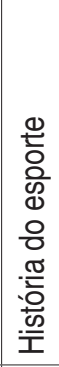 & 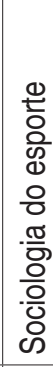 & $\begin{array}{l}\frac{0}{2} \\
\frac{1}{0} \\
0 \\
\frac{8}{0} \\
\frac{\pi}{0} \\
\frac{8}{0} \\
\frac{8}{0}\end{array}$ & $\begin{array}{l}\frac{\pi}{8} \\
0 \\
0\end{array}$ \\
\hline 2 & 0 & 0 & 1 & 1 \\
\hline 0 & 0 & 0 & 0 & 0 \\
\hline 0 & 0 & 0 & 0 & 0 \\
\hline 0 & 0 & 0 & 0 & 0 \\
\hline 0 & 0 & 0 & 0 & 0 \\
\hline 0 & 0 & 0 & 0 & 0 \\
\hline 0 & 0 & 0 & 0 & 0 \\
\hline 0 & 0 & 0 & 0 & 0 \\
\hline 0 & 0 & 0 & 0 & 0 \\
\hline 0 & 0 & 0 & 0 & 0 \\
\hline 0 & 0 & 0 & 0 & 0 \\
\hline 0 & 0 & 0 & 0 & 0 \\
\hline 0 & 0 & 0 & 0 & 0 \\
\hline 0 & 0 & 0 & 0 & 0 \\
\hline 1 & 0 & 0 & 1 & 0 \\
\hline 2 & 2 & 0 & 0 & 0 \\
\hline 0 & 0 & 0 & 0 & 0 \\
\hline 0 & 0 & 0 & 0 & 0 \\
\hline 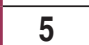 & 2 & 0 & 2 & 1 \\
\hline
\end{tabular}

Fonte: os autores.

\subsection{Idioma das publicações}

Analisar o idioma da escrita permite estimar 0 alcance das publicações científicas e ajuda a identificar a localização de polos de pesquisa ao redor do mundo.

Dos 105 artigos analisados, 67 (63,8\%) foram disponibilizados apenas em inglês, 30 $(28,6 \%)$ apenas em português, sete $(6,7 \%)$ em português e inglês e um $(1 \%)$ em inglês e espanhol. 0 Gráfico 1 expressa a distribuição por idioma das publicações através dos anos. 


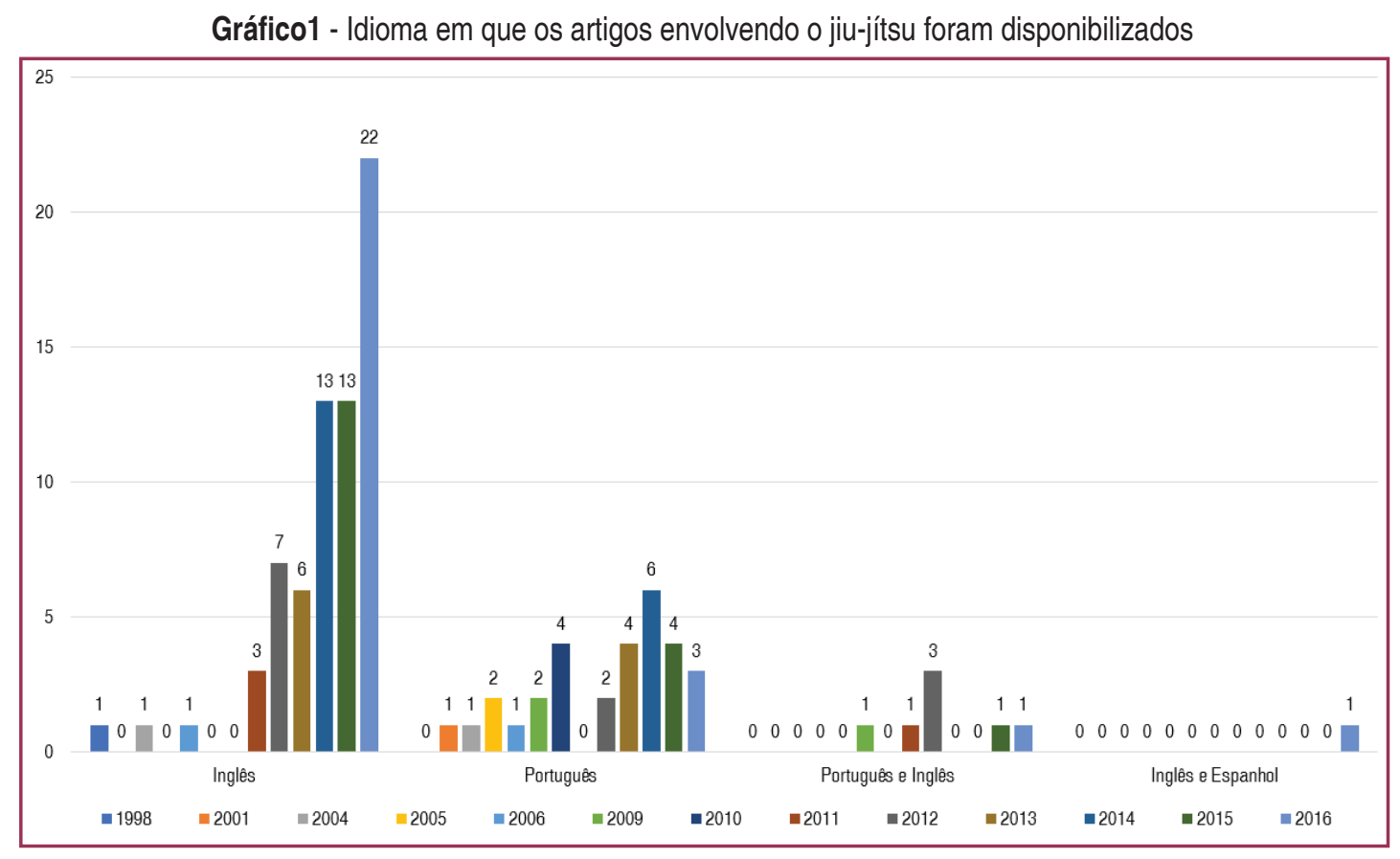

Fonte: os autores.

\subsection{Análise cronológica da pesquisa}

A análise do ano de publicação dos documentos estudados permitiu observar a distribuição cronológica da pesquisa com o jiu-jítsu. Vale ressaltar que as bases de dados também estão sujeitas à não indexação de trabalhos mais antigos. Todavia, os dados observados possibilitam, com maior precisão, a reflexão sobre o cenário da pesquisa envolvendo o jiu-jítsu nas duas últimas décadas. $O$ Gráfico 2 representa a distribuição cronológica da publicação de artigos comparada à de teses e dissertações no Brasil envolvendo o jiu-jítsu.

Gráfico 2 - Distribuição cronológica da publicação de artigos, teses e dissertações no Brasil envolvendo o jiu-jítsu.

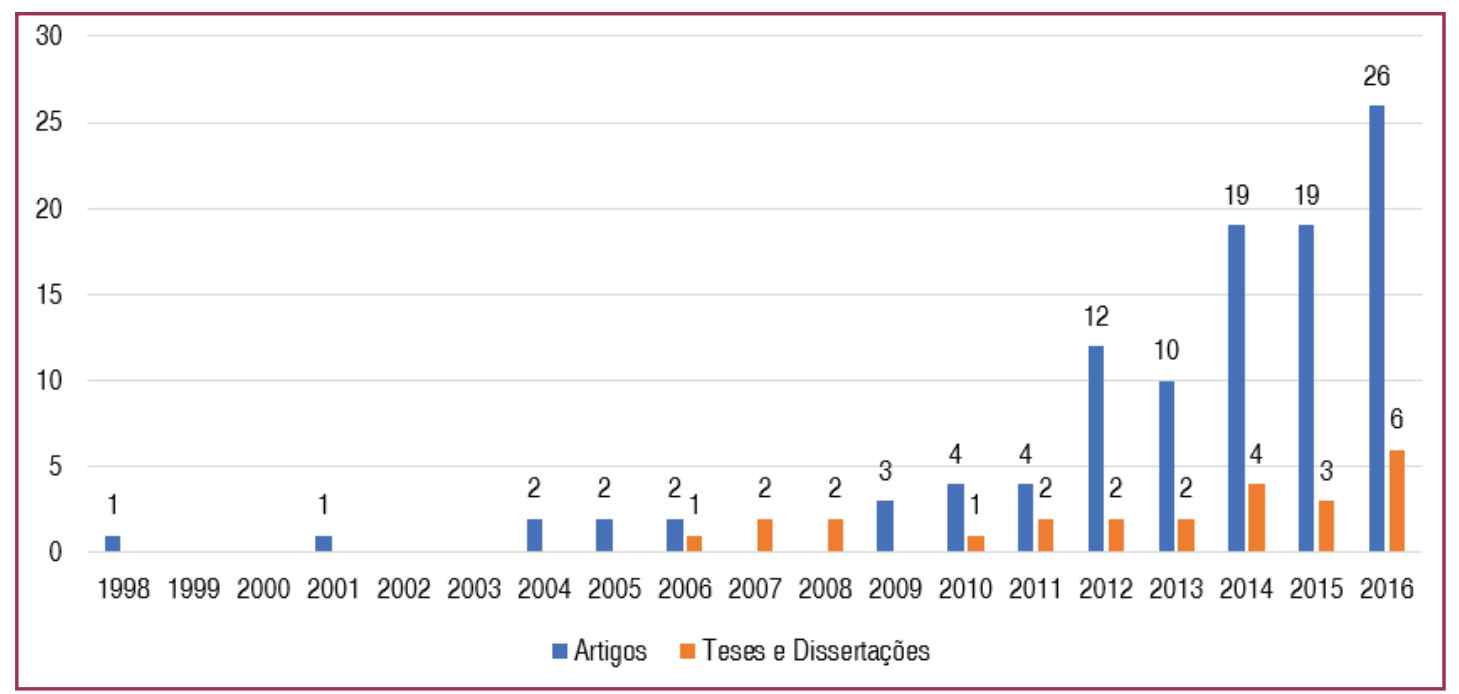

Fonte: os autores.

\section{DISCUSSÃO}

Tanto os artigos quanto as teses e dissertações brasileiras envolvendo o jiu-jítsu foram classificados de forma majoritária como pesquisas de caráter quantitativo. Para Creswell 
(2007), tal abordagem se baseia em redução de variáveis específicas, raciocínio de causaefeito e testes de hipóteses através de dados quantificáveis frutos da coleta. Em suma, 0 pesquisador já reconhece as variáveis que pretende estudar, o que implica a existência de um corpo de pesquisas prévio sobre 0 assunto.

Em menor quantidade, estão as pesquisas de caráter qualitativo e quantitativoqualitativo, nas quais o pesquisador analisa documentos históricos, imagens e filmagens, além dos discursos e ações de sujeitos ou grupos através de entrevistas, observação e interpretação, criando categorias de análise que podem vir a contribuir posteriormente com a investigação quantitativa (CRESWELL, 2007).

A mesma diferença observada entre as abordagens de pesquisa se mostra presente em relação às subáreas de pesquisa no estudo do jiu-jítsu. A maioria dos trabalhos se relaciona com a biodinâmica, em detrimento do menor número de trabalhos envolvendo a sociocultural e pedagógica. Essa diferença reflete como um fruto da caracterização da pós-graduação brasileira em Educação Física, em que a subárea biodinâmica concentra a maior parte do corpo docente e linhas de pesquisa (MANOEL; CARVALHO, 2011), enquanto a subárea sociocultural e pedagógica se situa na periferia do sistema nacional de pós-graduação da área 21 (CARNEIRO et al., 2016; GUTIERREZ; ALMEIDA; MARQUES, 2016).

A análise da disciplina de estudo dos trabalhos resultou em uma destacada diversidade dentro de cada subárea de pesquisa. Porém, observa-se uma distribuição desigual por abordagem e subárea de pesquisa. Estudos relacionados a "fisiologia do exercício", "traumatologia", "biomecânica" e "nutrição esportiva" compõem, juntos, a grande maioria. Já disciplinas pertencentes à subárea sociocultural e pedagógica, além do "esporte adaptado/ fisiologia", são pouco recorrentes na literatura sobre jiu-jítsu, guardando relação com os resultados expressos por Correia e Franchini (2009), ao analisarem literatura relacionada à L/ AM/MEC em periódicos brasileiros.

Dentre os diversos trabalhos da subárea biodinâmica, se destacam aqueles relacionados à fisiologia do exercício e, em especial, voltados a analisar as demandas fisiológicas impostas aos atletas em situação de combate (DIAZ-LARA et al., 2015). Alguns desses trabalhos também trazem contribuições pertinentes acerca de aspectos técnico-táticos (ANDREATO et al., 2016). Outra disciplina que se mostrou muito investigada foi a traumatologia, com estudos voltados à quantificação e classificação epidemiológica das lesões que acontecem em ambiente competitivo (KREISWIRTH; MYER; RAUH, 2014).

Na subárea sociocultural e pedagógica, alguns trabalhos contribuem para o entendimento de contextos históricos em que o jiu-jítsu se desenvolveu (LISE; SANTOS; CAPRARO, 2014). Diferentes contextos sociais são discutidos com base na Sociologia, como exemplo, a relação entre jiu-jítsu e violência (TEIXEIRA, 2010). Enquanto outros estudos foram identificados em menor número nas disciplinas de Psicologia e Pedagogia do esporte, nenhum foi relacionado à gestão esportiva.

Deste modo, questões técnico-táticas e tópicos relacionados à gestão esportiva, corroborando Franchini e Del Vecchio (2011), representam um terreno fértil para a pesquisa futura no jiu-jítsu, considerando o crescente número de competições e a falta de padronização de regras entre as várias federações que reivindicam a organização dessa modalidade esportiva (PAIVA, 2009). 
No que diz respeito aos participantes dos estudos envolvendo o jiu-jítsu, observa-se uma distribuição heterogênea composta por sujeitos envolvidos de diferentes formas com esta modalidade esportiva. 0 esporte contemporâneo é tido como fenômeno heterogêneo que pode assumir múltiplos sentidos, de acordo com os sujeitos envolvidos em sua prática, a modalidade e o ambiente em que esta se desenvolve (MARQUES; ALMEIDA. GUTIERREZ, 2007; MARQUES; GUTIERREZ; ALMEIDA, 2008). O fato de que a maioria dos trabalhos teve como participantes praticantes adultos não competidores permite destacar certo interesse por sujeitos envolvidos com o jiu-jítsu em ambientes diferentes do alto rendimento, enquanto uma menor parte das pesquisas foi realizada com indivíduos competidores. Um número reduzido de pesquisas contou como participantes os idosos, pessoas com deficiência, adolescentes, instrutores, professores e mestres, deixando em evidência algumas das lacunas na pesquisa com o jiu-jítsu.

Quanto ao gênero dos participantes, é discrepante a diferença entre o número de estudos com homens e com mulheres. A predominância masculina também se destaca quando comparada a estudos envolvendo ambos os gêneros. Essa diferença evidencia a baixa representação de mulheres em um universo predominantemente masculino, assim como no MMA (THOMAZINI, MORAES; ALMEIDA, 2008). Isto se dá devido a uma cultura de masculinidades "cascas-grossas" historicamente atrelada ao jiu-jítsu, que contribui para uma visível segregação de gênero (FERREIRA, 2016). Futuramente, importantes lacunas devem ser preenchidas com mais estudos envolvendo mulheres, sob a perspectiva de cada subárea e disciplina relacionada ao jiu-jítsu.

O Brasil, além de se destacar no cenário competitivo do jiu-jítsu, é o país onde se situa a maioria das instituições, grupos de pesquisa e pesquisadores publicando sobre a modalidade. A maior parte dos autores dos artigos é brasileira e a predominância de origem das teses e dissertações ocorre em instituições das regiões Sul e Sudeste do Brasil, onde se concentra a maior parte dos cursos de pós-graduação em Educação Física no país (GENTIL; BRITO NETO, 2015).

A maioria dos artigos sobre o jiu-jítsu foi classificada nos estratos Qualis A2 e B1, sendo relevante também o número de publicações com classificação A1. Brauer et al. (2014), ao analisarem publicações datadas entre 1998 e 2011, envolvendo L/AM/MEC, em 36 periódicos com classificação Qualis A1 e A2, com relação direta com a Educação Física, encontraram uma proporção semelhante ao observado no jiu-jítsu neste trabalho, no que diz respeito às subáreas de pesquisa. Porém, os autores não identificaram nenhuma publicação relacionada especificamente ao jiu-jítsu em periódicos com esta classificação (A1 e A2). Os dados do presente estudo evidenciam uma tendência de crescimento qualitativo da pesquisa no jiu-jítsu nos últimos anos.

Foi possível relacionar os periódicos em que publicações sobre jiu-jítsu são recorrentes a determinadas subáreas de pesquisa e disciplinas de estudo, permitindo a identificação de periódicos cujo escopo se relaciona com determinada subárea ou disciplina. A relação destes dados com o estrato Qualis de cada periódico permitiu observar que a maioria com classificação A1 e A2 com mais de dois artigos sobre o jiu-jítsu veicula estudos relacionados à subárea biodinâmica, talvez devido a uma maior oferta de possibilidades de publicação nesses estratos para esta subárea (GUTIERREZ, ALMEIDA, MARQUES, 2016). Em comparação à distribuição por subáreas de pesquisa, a sociocultural e pedagógica tem uma representação ainda menor, 
deixando evidente a pequena representatividade e inserção desta subárea de pesquisa no jiu-jítsu.

Segundo Forattini (1997), o inglês se consolida como língua franca da ciência, sendo o idioma das informações divulgadas pela maioria dos periódicos acadêmicos do mundo, 0 que não foi diferente com o jiu-jítsu. Porém, uma quantidade significativa de artigos analisados neste estudo está disponível apenas em português, destacando a relevância do Brasil na produção científica sobre essa modalidade esportiva. É possível considerar que os artigos em português não tenham a mesma visibilidade no cenário internacional em comparação aos artigos disponíveis em inglês. A análise das publicações através dos anos permitiu observar que a literatura acadêmica do jiu-jítsu está em processo de internacionalização, devido à tendência observada de crescimento no número de publicações em inglês nos últimos anos.

A análise cronológica dos estudos permite observar um crescimento acentuado da pesquisa com o jiu-jítsu a partir da década de 2010, quando passou a ser, a cada ano, mais explorado por acadêmicos. É possível relacionar o aumento na produção científica sobre o jiujítsu com o crescimento no número de praticantes e de visibilidade no cenário esportivo (PAIVA, 2009). Além disso, Gentil e Brito Neto (2015) relatam o crescimento no número de cursos de pós-graduação stricto sensu em Educação Física no Brasil na última década, o que impulsionou a produção científica do país. O crescimento das publicações acadêmicas sobre o jiu-jítsu é destacável e sugere que ainda não alcançou um processo de estagnação. Considerando a história da pesquisa no jiu-jítsu juntamente com o potencial das lacunas destacadas neste trabalho, estima-se franco crescimento do corpo de conhecimentos acadêmicos nos próximos anos.

\section{CONSIDERAÇÕES FINAIS}

Este estudo bibliométrico permitiu que, através da Análise Temática de artigos em periódicos acadêmicos e de teses e dissertações brasileiras envolvendo o jiu-jítsu, fosse possível analisar e descrever o cenário de pesquisa nesta modalidade esportiva, apontando lacunas e tendências. Deve-se reconhecer que esta não se trata da totalidade de material acadêmico desenvolvido, que conta também com livros, teses e dissertações não disponibilizadas para download pelo Portal Capes, trabalhos de conclusão de curso e comunicações em congressos, como exemplos.

É evidente que existe certa desigualdade numérica no que diz respeito à abordagem de pesquisa, subárea de pesquisa e disciplina de estudo, destacando-se a pesquisa quantitativa, com disciplinas de estudos relacionadas à subárea biodinâmica. Este levantamento permitiu observar lacunas passíveis de investigação futura, como disciplinas de estudo relacionadas à subárea sociocultural e pedagógica, além do esporte adaptado.

A caracterização dos participantes evidenciou o envolvimento heterogêneo com o jiujítsu por seus praticantes. Um número reduzido de pesquisas envolveu idosos, pessoas com deficiência, adolescentes, instrutores, professores e mestres, participantes que representam um terreno fértil para a pesquisa nos próximos anos. Também é pertinente a lacuna deixada pela substancial falta de estudos envolvendo mulheres, que se dá pela reduzida participação feminina no jiu-jítsu (FERREIRA, 2016). 
O Brasil, talvez por se tratar do país de origem do jiu-jítsu em suas configurações atuais, é também pioneiro e maior destaque na pesquisa acadêmica sobre essa modalidade esportiva. A maior parte das instituições e pesquisadores envolvidos na sua pesquisa se encontra no país, mas é perceptível a formação de polos de pesquisa em outros países.

A análise do idioma das publicações através dos anos mostra um crescimento no número de publicações em inglês, enquanto ainda é considerável o número de artigos disponíveis apenas em português, deixando evidente que, na esfera acadêmica, o jiu-jítsu segue os mesmos sentidos de internacionalização tomados no âmbito esportivo na última década. Também são apresentadas evidências de que os artigos são publicados cada vez mais em estratos Qualis superiores.

A análise cronológica permitiu identificar o crescimento destacável em curso na produção científica do jiu-jítsu a partir do início da década de 2010, quando se alcançou maior projeção internacional.

A partir destas observações, foi possível analisar e descrever um cenário sobre a produção científica em torno do jiu-jítsu no Brasil e no mundo, apontando para uma série de lacunas passíveis de investigação futura, tendo em vista que a pesquisa sobre esta modalidade esportiva se encontra em franca expansão mundial.

\section{REFERÊNCIAS}

ABREU, Teo Bueno; FERNANDES, João Paulo; MARTINS, Isabel. Uma análise qualitativa e quantitativa da produção cientifica sobre CTS (ciência, tecnologia e sociedade) em periódicos da área de ensino de ciências no Brasil. In: ENCONTRO NACIONAL DE PESQUISA EM EDUCAÇÃO EM CIÊNCIAS, 7, 2009. Atas.... Florianópolis, 2009. Disponível em: <http://www. posgrad.fae.ufmg.br/posgrad/viienpec/pdfs/852.pdf>. Acesso em: 2 janeiro 2019.

ANDREATO, Leonardo Vidal et al. Brazilian Jiu-Jítsu combat among different categories: timemotion and physiology. A systematic review. Strength \& Conditioning Journal, v. 38, n. 6, p. 44-54, 2016.

AQUINO, Rodrigo et al. A produção científica acerca do aspecto tático-técnico nos jogos esportivos coletivos: análise dos periódicos brasileiros. Movimento, v. 23, n. 4, p. 1407-1420, 2017.

ARAÚJO, Carlos Alberto. Bibliometria: evolução histórica e questões atuais. Em questão, v. 12, n. 1, p. 11-32, 2006.

BARATA, Rita de Cássia Barradas. Dez coisas que você deveria saber sobre o Qualis. Revista Brasileira de Pós-Graduação, v. 13, n. 30, p. 13-40, 2016.

BLEDSOE, Gregory et al. Incidence of injury in professional mixed martial arts competitions. Journal of sports science \& medicine, v. 5, p. 136-142, 2006.

BRAUER, André et al. Produção acadêmica em lutas, artes marciais e esportes de combate. Cadernos da Escola de Educação e Humanidades, v. 1, n. 7, p. 01-10, 2014. 
BRAUN, Virginia; CLARKE, Victoria. Using thematic analysis in psychology. Qualitative research in psychology, v. 3, n. 2, p. 77-101, 2006.

CARNEIRO, Felipe Ferreira Barros et al. Uma revista em movimento: contribuições para a subárea sociocultural e pedagógica da Educação Física brasileira (2004-2014). Movimento, v. 22, n. 1, 2016.

COORDENAÇÃO DE APERFEIÇOAMENTO DE PESSOAL DE NÍVELSUPERIOR CAPES. Critérios de classificação Qualis área 21. Brasília, 2016. Disponível em: <https:// www.capes.gov.br/images/documentos/Qualis periodicos 2016/CRIT\%C3\%89RIOS DE CLASSIFICA $\%$ C3 $\% 87 \%$ C3\%830 QUALIS EDUCA $\% C 3 \% 87 \%$ C3\% 830 F $\%$ C3\%8DSICA.pdf $>$. Acesso em: 17 maio 2018.

COORDENAÇÃO DE APERFEIÇOAMENTO DE PESSOAL DE NÍVEL SUPERIOR - CAPES. WEBQUALIS CAPES. Disponível em: <https://sucupira.capes.gov.br/sucupira/public/consultas/ coleta/veiculoPublicacaoQualis/listaConsultaGeralPeriodicos.jsf >. Acesso em: 17 maio 2018.

CORREIA, Walter Roberto; FRANCHINI, Emerson. Produção acadêmica em lutas, artes marciais e esportes de combate. Motriz, v. 16, n. 1, p. 01-09, 2009.

CRESWELL, John W. Projeto de Pesquisa: métodos qualitativo, quantitativo e misto. 2. ed. Porto Alegre: Artmed, 2007.

DIAZ-LARA, Francisco Javier et al. Analysis of physiological determinants during an international Brazilian Jiu-jítsu competition. International Journal of Performance Analysis in Sport, v. 15, n. 2, p. 489-500, 2015.

FERREIRA, Douglas. As culturas do jiu-jítsu e a produção de corpos e de masculinidades “cascas-grossas". 2016. 118f. Dissertação (Mestrado) - Programa de Pós-Graduação em Educação, Universidade Luterana do Brasil, Canoas, 2016.

FERREIRA, Norma Sandra de Almeida. As pesquisas denominadas "estado da arte". Educação e sociedade, v. 23, n. 79, p. 257-272, 2002.

FORATTINI, Oswaldo Paulo. A língua franca da ciência. Revista de Saúde Pública, v. 31, n. 1, p. 3-8, 1997.

FRANCHINI, Emerson; DEL VECCHIO, Fabrício Boscolo. Estudos em modalidades esportivas de combate: estado da arte. Revista Brasileira de Educação Física e Esporte, v. 25, n. especial, p. 67-81, 2011.

GENTIL, Raphael do Nascimento; BRITO NETO, Aníbal. A expansão da pós-graduação em educação física no Brasil. In: CONGRESSO BRASILEIRO DE CIÊNCIAS DO ESPORTE, 19. 2015.,. Anais...Vitória, 2015. v. 9. Disponível em: <http://congressos.cbce.org.br/index.php/ conbrace2015/6conice/paper/viewFile/7607/3410>. Acesso em: 2 janeiro 2019.

GREEN, Thomas; SVINTH, Joseph. Martial Arts of the World: an encyclopedia of history and innovation. Santa Barbara: ABC-CLIO, 2010.

GUTIERREZ, Gustavo Luis; ALMEIDA, Marco Antonio Bettine de; MARQUES, Renato Francisco Rodrigues. Apropriação das ciências humanas pela Educação Física: análise dos processos de classificação no Brasil entre os anos de 2007 e 2012. Revista Brasileira de Educação Física e Esporte, v. 30, n. 4, p. 937-949, 2016. 
INTERNATIONAL BRAZILIAN JIU-JITSSU FEDERATION. IBJJF World Map. Disponível em: $<$ http://ibjjf.com/ibjjf-world-map/ >. Acesso em: 15out. 2018.

KREISWIRTH, Ethan; MYER, Gregory; RAUH, Mitchell. Incidence of injury among male Brazilian jiu-jitsu fighters at the World Jiu-Jítsu No-Gi Championship 2009. Journal of athletic training, v. 49, n. 1, p. 89-94, 2014.

LISE, Riqueldi Straub; SANTOS, Natasha; CAPRARO, André Mendes. "A legenda dos Gracie": uma análise da crônica de Nelson Rodrigues. Movimento, v. 20, n. 4, p. 1329-1349, 2014.

MANOEL, Edison de Jesus; CARVALHO, Yara Maria de. Pós-graduação na educação física brasileira: a atração (fatal) para a biodinâmica. Educação e Pesquisa, v. 37, n. 2, p. 389-406, 2011.

MARQUES, Renato Francisco Rodrigues; ALMEIDA, Marco Antonio Bettine; GUTIERREZ, Gustavo Luis. Esporte: um fenômeno heterogêneo: estudo sobre o esporte e suas manifestações na sociedade contemporânea. Movimento, v. 13, n. 3, p. 225-242, 2007.

MARQUES, Renato Francisco Rodrigues; GUTIERREZ, Gustavo Luis; ALMEIDA, Marco Antonio Bettine. $O$ esporte contemporâneo e o modelo de concepção das formas de manifestação do esporte. Conexões, v. 6, n. 2, p. 42-61, 2008.

MATOS-SOUZA, José R. et al. Impact of Adapted Sports Activities on the Progression of Carotid Atherosclerosis in Subjects With Spinal Cord Injury. Archives of physical medicine and rehabilitation, v. 97, n. 6, p. 1034-1037,2016.

MINAYO, Maria Cecilia; SANCHES, Odécio. Quantitativo-qualitativo: oposição ou complementaridade? Cadernos de saúde pública, v. 9, n. 3, p. 237-248, 1993.

PAIVA, Leandro. Pronto Pra Guerra: Preparação física específica para luta e superação. Manaus: OMP Editora, 2009.

TEIXEIRA, Antonio Claudio Engelke Menezes. Sobre Lutadores e "Pitboys": A experiência da violência entre jovens de classe média e alta do Rio de Janeiro. Cadernos de Segurança Pública, v. 1, p. 3-13, 2010.

THOMAS, Jerry; NELSON, Jack; SILVERMAN, Stephen. Métodos de pesquisa em atividade física. 5. ed. Porto Alegre: Artmed, 2007.

THOMAZINI, Samuel Oliveira; MORAES, Cláudia Emília Aguiar; ALMEIDA, Felipe Quintão. Controle de si, dor e representação feminina entre lutadores(as) de mixed martial arts. Pensar a Prática, v. 11, n. 3, p. 281-290, 2008.

VICENTINI, Lucas; MARQUES, Renato Francisco Rodrigues. A carreira profissional no jiu-jítsu brasileiro: A perspectiva de atletas de alto rendimento que atuam como professores. In: PAIVA, Leandro. Ciências Aplicada às artes marciais. 2. ed. Manaus: OMP Editora, 2018. p. 122. 
